\title{
Pharmacist-led medication non-adherence intervention: reducing the economic burden placed on the Australian health care system
}

This article was published in the following Dove Press journal: Patient Preference and Adherence

\author{
Rachelle Louise Cutler' \\ Andrea Torres-Robles' \\ Elyssa Wiecek' \\ Barry Drake ${ }^{2}$ \\ Naomi Van der Linden ${ }^{3}$ \\ Shalom I (Charlie) Benrimoj ${ }^{4}$ \\ Victoria Garcia-Cardenas' \\ 'Graduate School of Health, University of \\ Technology Sydney, Sydney, NSW, \\ Australia; ${ }^{2}$ Faculty of Engineering and \\ Information Technology, University of \\ Technology Sydney, Sydney, NSW, \\ Australia; ${ }^{3}$ AstraZeneca Netherlands, \\ The Hauge, Netherlands; ${ }^{4}$ Sydney \\ University, Sydney, NSW, Australia
}

Background: Scarcity of prospective medication non-adherence cost measurements for the Australian population with no directly measured estimates makes determining the burden medication non-adherence places on the Australian health care system difficult. This study aims to indirectly estimate the national cost of medication non-adherence in Australia comparing the cost prior to and following a community pharmacy-led intervention.

Methods: Retrospective observational study. A de-identified database of dispensing data from 20,335 patients $(n=11,257$ on rosuvastatin, $n=6,797$ on irbesartan and $n=2,281$ on desvenlafaxine) was analyzed and average adherence rate determined through calculation of PDC. Included patients received a pharmacist-led medication adherence intervention and had twelve months dispensing records; six months before and six months after the intervention. The national cost estimate of medication non-adherence in hypertension, dyslipidemia and depression pre- and post-intervention was determined through utilization of disease prevalence and comorbidity, non-adherence rates and per patient disease-specific adherencerelated costs.

Results: The total national cost of medication non-adherence across three prevalent conditions, hypertension, dyslipidemia and depression was $\$ 10.4$ billion equating to $\$ 517$ per adult. Following enrollment in the pharmacist-led intervention medication non-adherence costs per adult decreased \$95 saving the Australian health care system and patients $\$ 1.9$ billion annually.

Conclusion: In the absence of a directly measured national cost of medication nonadherence, this estimate demonstrates that pharmacists are ideally placed to improve patient adherence and reduce financial burden placed on the health care system due to nonadherence. Funding of medication adherence programs should be considered by policy and decision makers to ease the current burden and improve patient health outcomes moving forward.

Keywords: medication adherence, community pharmacy, big data, dispensing records, health economics

\section{Introduction}

Appropriate use of medications remains sub-optimal despite their proven effectiveness in preventing and managing chronic conditions. ${ }^{1}$ In an outpatient setting medication non-adherence is one of the principal obstacles in successful pharmacotherapy, yet often fails to be clinically recognized. ${ }^{1}$ The high prevalence of medication non-adherence is associated with increased morbidity and mortality, disease progression and increased utilization of health care resources and
Correspondence: Rachelle Louise Cutler Graduate School of Health, University of Technology Sydney, PO Box 123 ,

Broadway, Sydney, NSW 2007, Australia

Tel +61295147187

Email rachelle.cutler@uts.edu.au 
accompanying expenditure. ${ }^{2,3}$ Nevertheless medication non-adherence remains a neglected element of patient therapeutic management.

Internationally the economic impact of medication non-adherence has been examined at the macro-level in a number of studies, independent reports and gray literature findings. Heterogeneity exists in the reported costs with limited information available to determine how these values were calculated. The 2013 IMS "Avoidable Costs in US Healthcare" report stipulates that annually US\$105.4 billion or $3.9 \%$ of the nation's health care spending would be avoidable if medication nonadherence were addressed. ${ }^{4}$ Furthermore, the "Advancing the responsible use of medicines" report specifies that medication non-adherence contributes $57 \%$ of the world's total avoidable cost due to suboptimal medicines use. ${ }^{5}$ The quantification of cost avoidance and the research substantiating these analyses implies that better use of medicines can improve quality of life through reduced hospitalizations and improved health outcomes such as morbidity and mortality. Figure 1 presents a timeline of the global predictive annual economic burden attributed to medication non-adherence reported in the gray literature, highlighting the tendency of medication non-adherence costs to increase over time.

The microlevel economic examination of medication non-adherence within single disease state studies supports the determination that non-adherence is largely associated with higher health care costs ${ }^{2,6-9}$ and solidifies big data international projections. ${ }^{4,10} \mathrm{~A}$ recent systematic review reported the annual adjusted disease-specific economic cost of non-adherence to range from US\$949 to $\$ 44,190$ per person. Costs associated with non-adherence to cancer treatment $(\$ 114,101)$ were substantially higher than costs associated with non-adherence to treatment for cardiovascular disease $(\$ 16,124)$, mental health $(\$ 16,110)$ or osteoporosis $(\$ 43,240) .^{11}$ These micro costing studies, however, are limited as they only report for a specific population and fail to take into consideration recent changes in disease prevalence. Additionally, the majority of the studies are conducted in the United States (US) where health care is generally more expensive, and the health insurance system differs significantly between the US and Australia. Scarcity of prospective medication non-adherence cost measurements for the Australian population with no directly measured estimates reported makes the generalisability of these

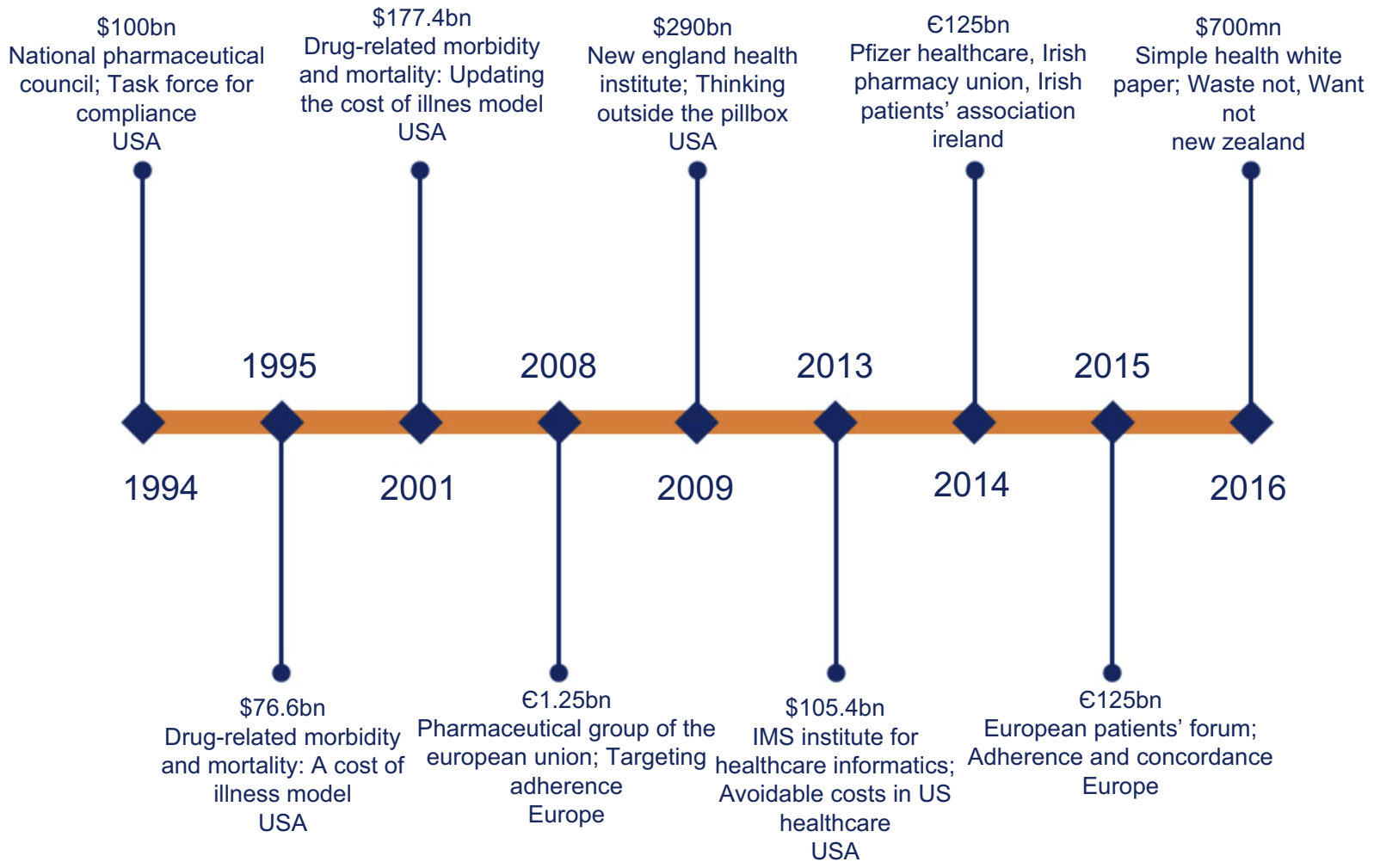

Figure I Timeline of macro-level medication non-adherence costs. Gray literature reports data demonstrating the increasing costs associated with medication nonadherence over time.

Note: Costs in $\$$ are expressed in US\$. 
results limited in their pertinence to extrapolating national cost estimates of medication non-adherence.

In Australia, inappropriate use of medicines costs the Australian public hospital system AUD $\$ 1.2$ billion per year representing $2-3 \%$ of all hospital admissions, with this figure rising to $20-30 \%$ of all admissions in the population aged 65 years and over. ${ }^{12}$ It is estimated that $4.7 \%$ of the total Australian health expenditure is avoidable due to suboptimal medicines use ${ }^{5}$ extrapolating to AUD\$8 billion annually. ${ }^{13}$ These costs, however, do not directly estimate medication non-adherence and take into consideration a number of confounding factors that contribute to inappropriate medication use. In addition to medication nonadherence, these avoidable costs arise when patients fail to receive the right medications at the right time or in the right way, or receive them but fail to take them. Medication nonadherence has been identified as an "opportunity cost" to reclaim current health care spending wastage. ${ }^{4}$ Targeted analysis is required to accurately estimate the associated cost of medication non-adherence in Australia, which is thought to be significantly underestimated. ${ }^{14-16}$

Improvements in health care in conjunction with prolonged life expectancy has resulted in a rise in the prevalence of chronic conditions ultimately increasing the burden placed on health care systems and subsequently leading to a higher number of prescription medications and budgetary spending allocations to manage these conditions. ${ }^{17}$ Most illnesses and deaths in Australia are caused by chronic conditions with an estimated 1 in 2 Australians (50\%) suffering from at least one chronic condition. ${ }^{18}$ Cardiovascular disease and mental health conditions are two of the most prevalent chronic conditions, with 1 in 5 (18\%) Australians experiencing one of these. ${ }^{18}$ Suboptimal adherence to commonly prescribed medications in cardiovascular disease and mental health contribute significantly to disease progression and mortality, ${ }^{19-22}$ increasing the budget impact on the Australian health care system. Increased availability of large prescription data sets enhances the analysis and evaluation of patient medication adherence, enabling a cost-effective approach to estimate the economic impact of medication nonadherence. $^{23,24}$

With up to $30 \%$ of the prescriptions never being filled and approximately $50 \%$ of the people with chronic conditions stopping their medications within the first twelve months ${ }^{25}$ the negative financial implications of medication non-adherence are of paramount concern. Evidence supports that community pharmacists are ideally situated to deliver medication adherence interventions,${ }^{26}$ however, further examination is necessary to determine the broader economic impact pharmacist medication adherence interventions have in cost savings to the Australian health care system. In the absence of a directly measured national cost of medication non-adherence, this study aims to indirectly estimate the national cost of medication non-adherence in Australia comparing the cost prior to and following a community pharmacy-led intervention. Utilizing population-based and pharmacy claims data, a transparent and replicable model will be developed to determine the national estimate of medication non-adherence in hypertension, dyslipidemia and depression ${ }^{27}$ prior to and following a community pharmacy-based intervention through examining medication use of three molecules rosuvastatin, irbesartan and desvenlafaxine.

\section{Methods}

\section{Study design and data sources}

A retrospective analysis of de-identified patient pharmacy dispensing data from the GuildLink Pty Ltd database was conducted. GuildLink Pty Ltd is a wholly owned subsidiary of the Pharmacy Guild of Australia, which focuses on providing software solutions to community pharmacies to aid in the provision and documentation of pharmacy services.

The MedScreen Compliance program was utilized to identify patients receiving an educational-based intervention to enhance medication adherence from community pharmacies across Australia. This program is designed to help ensure that quality use of medicine is achieved and adherence to prescribed therapy is maintained or improved. The service targets non-adherent patients when a calculated medication possession ratio (MPR) is below $70 \%$. The clinical service consists of 1) identifying patient-specific barriers and facilitators to medication adherence; 2) engaging patients in a brief pharmacist educational interaction regarding adherence and quality use of medicines, this includes provision of either oral or written communication to enhance patient understanding and emphasize the importance of adherence; 3) goal setting for patient treatment targets and 4) recording the interaction and making patient-specific notes. ${ }^{28}$ Patients could receive one or multiple interventions across time periods depending on the calculated MPR, alerting the pharmacist to invite the patient to the intervention if they remain below the $70 \%$ adherence threshold. 
The unique patient identifier allowed patients enrolled in the MedScreen Compliance intervention to be anonymously identified and their medication adherence rates tracked. One year dispensing history was analyzed for each patient who completed the intervention; six months prior to the adherence intervention and six months following the intervention. Process indicators to validate the fidelity of the intervention were not available.

\section{Medication adherence}

Descriptive outcomes included adherence measures in patients with a dispensing history during the six months prior to the MedScreen Compliance intervention (pre-intervention/baseline), when the intervention was performed and six months following the intervention (post-intervention). For this analysis, adherence to three molecules rosuvastatin, irbesartan and/ or desvenlafaxine was determined using the proportion of days covered (PDC). The three molecules modeled disease state prevalence. PDC was defined as the total number of days supplied with the medication during the six-month period before and after the intervention divided by the total number of days in the fixed period. Analysis was conducted per trimesters, 6 months before and 6 months after the first pharmacist intervention, calculating the average PDC (\%) and standard deviation (SD) for all patients in each period using descriptive statistics. ${ }^{29}$ Adherence was defined as a PDC of $80 \%$ or greater, the most common threshold for adequate adherence to chronic medications. ${ }^{30}$ The number of nonadherent patients for each condition was determined by multiplying the rate of non-adherence pre- and post-intervention with the Australian adult population with the disease. This indicator was selected instead of MPR as it does not overestimate adherence, provides a conservative estimate and accounts for overlapping days supply. ${ }^{31}$

Estimates of the Australian population and number of patients with hypertension, dyslipidemia and depression were collected from the Australian Bureau of Statistics. ${ }^{18,32-35}$ The steps and sources of data used in the cost estimation are depicted in Figure 2 adapted from Nasseh et $\mathrm{al}^{27}$, data input values are presented in Table 1 . The prevalence rates of the conditions were multiplied by the total Australian population to determine national estimates.

\section{Cost calculations}

Monetary values attributed to medication non-adherence for hypertension, dyslipidemia and depression were identified from the literature. ${ }^{11}$ All costs were converted to

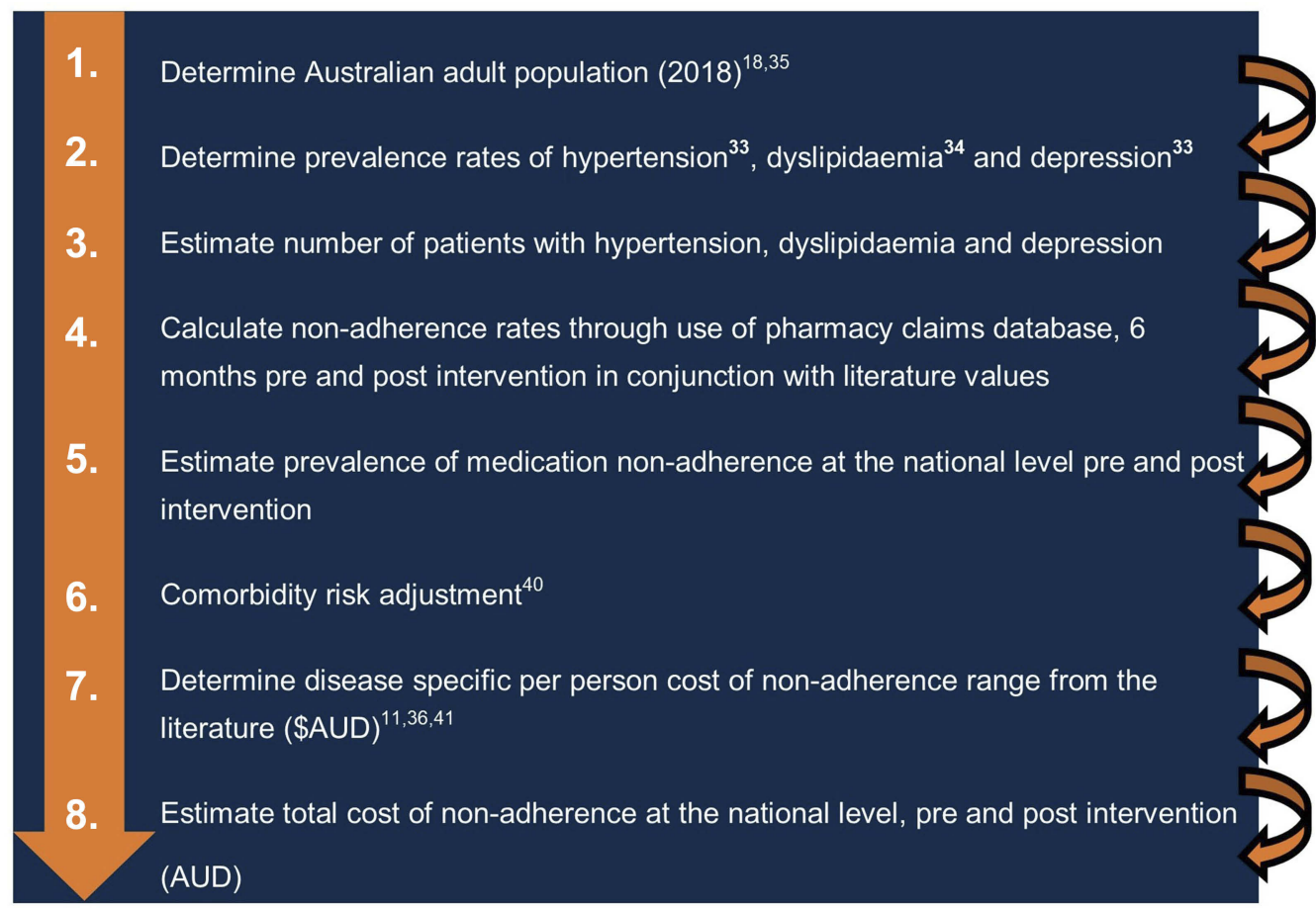

Figure 2 Derivation of the cost of medication non-adherence.

Note: Stepwise approach in the methodology (adapted from Nasseh et al ${ }^{23}$ ) undertaken to estimate the national cost of medication non-adherence in Australia pre- and post-community pharmacist-led intervention. 
Table I Derivation of cost process. Outlines data input values to determine the national cost estimate of medication non-adherence in Australia

\begin{tabular}{|c|c|c|c|c|c|c|}
\hline Derivation of cost process & \multicolumn{6}{|c|}{ Reported findings } \\
\hline Australian adult population $(2018)^{18,35}$ & \multicolumn{6}{|c|}{$20,160,000$} \\
\hline & \multicolumn{2}{|c|}{ Hypertension $^{33}$} & \multicolumn{2}{|c|}{ Dyslipidemia $^{34}$} & \multicolumn{2}{|c|}{ Depression $^{33}$} \\
\hline Prevalence rates of conditions & \multicolumn{2}{|l|}{$34 \%$} & \multicolumn{2}{|c|}{$32.8 \%$} & \multicolumn{2}{|c|}{$17.5 \%$} \\
\hline Prevalence rates of conditions (number of patients) & \multicolumn{2}{|c|}{$6,854,400$} & \multicolumn{2}{|c|}{$6,612,480$} & \multicolumn{2}{|c|}{$3,528,000$} \\
\hline \multirow[t]{2}{*}{ Average estimated PDC 6 month's pre- and post-intervention } & Pre & $45.5 \%$ & Pre & $45.6 \%$ & Pre & $52 \%$ \\
\hline & Post & $37.1 \%$ & Post & $37.6 \%$ & Post & $40.5 \%$ \\
\hline \multirow[t]{2}{*}{ National prevalence of medication non-adherence } & Pre & $3,118,752$ & Pre & $3,015,290$ & Pre & $1,834,560$ \\
\hline & Post & $2,542,982$ & Post & $2,486,292$ & Post & $\mathrm{I}, 428,840$ \\
\hline \multirow[t]{2}{*}{ Disease-specific non-adherence cost $(\$ A \cup D)^{11,36,41}$} & Min & $\$ 2,386$ & Min & $\$ 8,125$ & Min & $\$ 3,812$ \\
\hline & $\operatorname{Max}$ & $\$ 13,493$ & $\operatorname{Max}$ & $\$ 14,63 \mid$ & $\operatorname{Max}$ & $\$ 24,717$ \\
\hline \multirow[t]{2}{*}{ Conservative national estimate (\$AUD) } & Pre & $\$ 1,994,279,728$ & Pre & $\$ 6,565,793,975$ & Pre & $\$ 1,874,215,848$ \\
\hline & Post & $\$ 1,626,104,753$ & Post & $\$ 5,4 \mid 3,900,830$ & Post & $\$ 1,459,725,805$ \\
\hline \multirow[t]{2}{*}{ Radical national estimate (\$AUD) } & Pre & $\$ 11,277,793,957$ & Pre & $\$ 1|, 823,277,74|$ & Pre & $\$ 12,|52,4||, 63|$ \\
\hline & Post & $\$ 9,|95,738,24|$ & Post & $\$ 9,749,019,451$ & Post & $\$ 9,464,859,059$ \\
\hline
\end{tabular}

Abbreviations: PDC, proportion of days covered; Pre, pre-adherence intervention; Post, post-adherence intervention.

Australian dollars (2018 values) using the Cochrane Economics Methods Group - Evidence for Policy and Practice Information and Coordinating - Centre Cost Converter tool. ${ }^{36}$ The most conservative non-adherence cost estimate (minimum reported value) was utilized to extrapolate national expenditure attributed to nonadherence across each disease, radical estimates represented the maximum reported value in the literature. Comorbidity risk adjustment was undertaken to prevent duplication of non-adherence costs across multiple conditions. Within the non-adherent hypertension, dyslipidemia and depression population estimations were made for the number of patients with only 1 of the conditions and all combinations of 2 or 3 of the comorbid conditions. The national non-adherence cost estimate was further evaluated in terms of the cost outcome indicators that contributed to the total cost through application of the MACE framework ${ }^{37}$ examining national hospital cost data, ${ }^{38}$ and the Pharmaceutical Benefits Scheme expenditure data. ${ }^{39}$

\section{Sensitivity analysis}

A sensitivity analysis was conducted to quantify the changes in estimated total cost of non-adherence when varying adherence thresholds and cost inputs. As varying evidence exists quantifying the range of medication non-adherence rates, we conducted a sensitivity analysis using various adherence thresholds. For this sensitivity analysis in accordance with Meichenbaum et al, thresholds of $0.30,0.40,0.50,0.60$ in addition to pre-intervention and post-intervention thresholds identified in this study were analysed holding all other inputs fixed. ${ }^{40}$ Additional analyses were conducted to examine the effects of conservative versus radical cost inputs to estimations at $30 \%$ and $50 \%$ non-adherence rates.

\section{Ethical considerations}

Ethics approval was granted by the University of Technology Sydney Human Research Ethics Committee (approval number ETH18-2312).

\section{Results}

\section{Study population}

The GuildLink Pty Ltd database comprised prescription dispensing histories and intervention offerings for 2,530,562 million patients serviced through 3,318 pharmacies across Australia, providing in excess of 22 million dispensing records. A total of 20,335 patients $(n=11,257$ rosuvastatin, 
$\mathrm{n}=6,797$ irbesartan and $\mathrm{n}=2,281$ desvenlafaxine) from 1,805 pharmacies across Australia, were included in the analysis. The average number of patients per pharmacy was 11.27 (SD: 3.77) compared to 8.59 (SD: 5.14) across the entire database as patients visited multiple pharmacies.

The average age was highest in patients taking irbesartan 67 (SD: 12.42), followed by 65 (SD: 11.76) in rosuvastatin and 50 (SD: 15.70) for desvenlafaxine. Female patients represented a higher proportion of the population with the distribution of gender following a similar trend across molecules. For patients taking rosuvastatin $56 \%$ were female and $44 \%$ male, irbesartan $61 \%$ female and $39 \%$ male and desvenlafaxine $70 \%$ female and $30 \%$ male.

\section{Medication adherence}

The MedScreen Compliance intervention increased average PDC from 52.3\% at baseline (SD: 31.4 ) by $9.3 \%$ to $61.6 \%$ (SD: 31.7 ), while from the intervention time point average PDC increased from 49.3\% (SD: 30 ) by $12 \%$ to $61.6 \%(\mathrm{SD}: 31.7)$. Desvenlafaxine displayed the overall lowest average PDC of the three molecules at baseline (48\%, SD: 30.3) and postintervention (59.5\%, SD: 30.6$)$ however simultaneously demonstrated the greatest proportional increase in adherence over time following the intervention (PDC 11.5\%, SD: 29.3). Similar results were established between rosuvastatin and irbesartan with PDC over time increasing on average $8 \%$ (SD: 30.8 ) and $8.4 \%$ (SD: 31$)$ respectively. Across all three molecules there was a trend for medication adherence to decrease from baseline to the intervention, peak in the trimester following the intervention before slowly decreasing and plateauing (rosuvastatin PDC 62.4\% (SD: 31.7), irbesartan PDC 62.9\% (SD: 32) and desvenlafaxine PDC 59.5\% (SD: 30.6)).

\section{Cost estimation}

The total national cost of medication non-adherence across three prevalent disease states in 2018 prior to a community pharmacy-led intervention was $\$ 10.4$ billion equating to approximately $\$ 517$ per adult in Australia. Following enrollment in the MedScreen Compliance intervention medication non-adherence costs per adult decreased $\$ 95$ saving the Australian health care system and patients $\$ 1.9$ billion annually. Figure 3 depicts the national cost range of medication non-adherence pre and post-adherence intervention. Significant reductions in cost expenditure were demonstrated across all three conditions with depression exhibiting the greatest saving as a proportion of the original expenditure (22\%). Dyslipidaemia demonstrated the largest dollar figure saving ( $\$ 1.1$ billion), however, was the smallest as a proportion of the original expenditure $(17.5 \%)$. The same trends were demonstrated when applying the conservative and radical approach.

With over $85 \%$ of the non-adherence costs attributed to medical-related expenses, application of the MACE framework $^{37}$ to the conservative extrapolated costs facilitates the estimation of the proportion of costs attributed to various cost outcome indicators. Of the $\$ 8.4$ billion annual non-adherence cost post intervention, $\$ 2.1$ billion arose from the outpatient setting, $\$ 1.9$ billion from inpatient-related expenses, $\$ 1.8$ billion on prescription medications and $\$ 1.6$ billion were attributed to medical related costs such as general practitioner visits. If no adherence intervention is received the baseline nonadherence cost of $\$ 10$ billion can be broken down into

National cost range of medication non-adherence pre- and post- adherence intervention

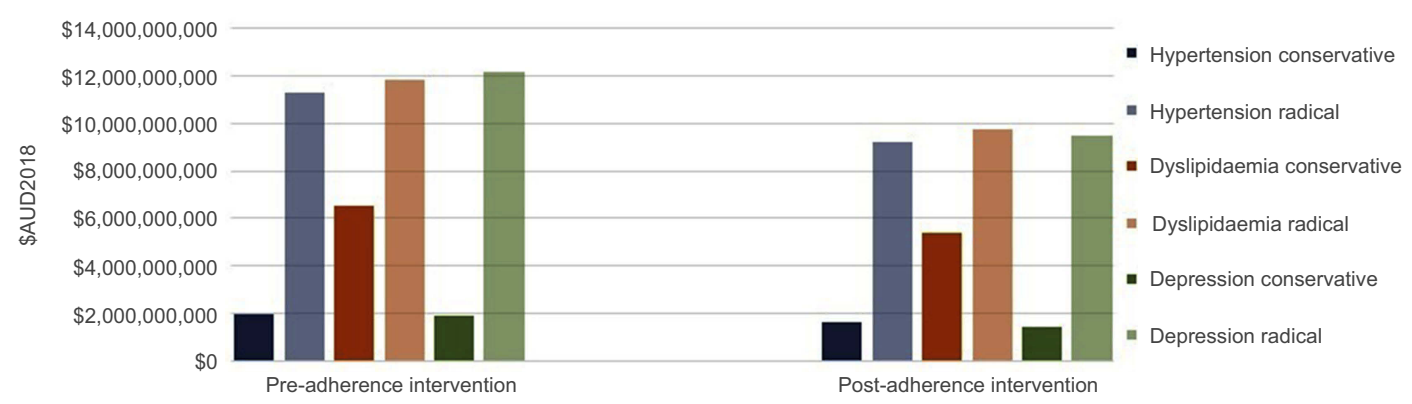

Figure 3 National cost range of medication non-adherence pre and post-adherence intervention. Bars represent the conservative and radical cost associated with medication non-adherence across three chronic conditions hypertension, dyslipidemia and depression. Chart comparison demonstrates Australian national cost range pre and post-community pharmacist-led medication adherence intervention. 
\$2.6 billion outpatient costs, \$2.3 billion inpatient costs, $\$ 2.2$ billion prescription medication and $\$ 1.9$ billion medical expenses.

\section{Sensitivity analysis}

Similar results were seen when cost estimation was performed using different adherence thresholds. Non-adherence costs decreased across all three molecules as adherence thresholds increased. Application of the conservative estimation across $30 \%$ and $50 \%$ non-adherence thresholds resulted in a total cost range varying between $\$ 6.7$ billion ( $\$ 333$ per adult) to $\$ 11.1$ billion ( $\$ 555$ per adult) annually. When applying the radical costing assumption to estimate disease-specific costs, the estimated national cost of medication non-adherence rose substantially ranging from $\$ 22.2$ billion $(\$ 1,102$ per adult) to $\$ 37$ billion ( $\$ 1,837$ per adult).

\section{Discussion}

The Australian national cost estimate of medication nonadherence across three highly prevalent chronic conditions prior to a community pharmacy-led intervention was $\$ 10.4$ billion or $\$ 517$ per adult. Aitken et $\mathrm{al}^{5}$ approximated that $4.7 \%$ of the total Australian health expenditure is avoidable due to suboptimal medicines use extrapolating to $\$ 8$ billion annually. ${ }^{13}$ This estimate did not directly measure medication non-adherence but rather examined inappropriate medication use. The extrapolated estimate although $25 \%$ higher than the value predicted by Aitken et al provides a more accurate evaluation utilizing pharmacy claims data, literature values and current disease prevalence to determine the cost of medication non-adherence in Australia. This cost reduced to $\$ 8.4$ billion or $\$ 421$ per adult following the MedScreen Compliance intervention.

Sustainability of the Australian health care system presents a major challenge and concern, with current levels of funding set to create significant financial burden for governments in the future. ${ }^{41}$ The emergence of new health challenges, the aging population and the increase in risk factors for chronic conditions in combination with the expectation of the Australian population to provide higher standards of care and subsidization fuel this situation. While Australia has a good health system by international standards, it is estimated that spending on health care by government as a percentage of gross domestic product will nearly double by $2050 .^{42}$ There are fears that the current level of funding is not sustainable and new models need to be considered. ${ }^{41}$ Subsidization of the Pharmaceutical Benefits Scheme represents $30 \%$ of the funds administered by the Department of Health and the Medical Benefits Scheme $52 \% .{ }^{43}$ Funding of strategies including pharmacist-led services to improve medication adherence, removes wastage and inefficient usage of the current system, resulting in more sustainable, cost-effective resource allocation.

Interventions to improve medication adherence have consistently demonstrated an improvement in health care outcomes and a reduction in total health care costs. ${ }^{44}$ A recent systematic review conducted by Milosavljevic et $\mathrm{al}^{45}$ found that overwhelmingly community pharmacistled intervention improved patients' adherence contributing to better blood pressure control, cholesterol management, chronic obstructive pulmonary disease and asthma control. However, studies in the review did not report statistically significant effects of interventions on diabetes or depression control, ${ }^{45}$ challenging the results found from improvements demonstrated with the MedScreen compliance intervention in depression. Data from the GuildLink Pty Ltd database supports the improvement of patient adherence rates in hypertension, dyslipidemia and depression measured through calculation of PDC increasing on average $9.3 \%$ from baseline and $12 \%$ from intervention date. Accompanying cost extrapolation data further harnesses these findings with a $\$ 1.9$ billion annual cost saving attributable to the MedScreen Compliance intervention across the three molecules. While the intervention demonstrates improvements in medication adherence rates in the six months ensuing, sustaining improvements long term requires further investigation and represents an obstacle in reducing future preventable health care expenditure.

The low number of studies directly comparing adherence interventions thwarts the determination of the most effective intervention. A recent network meta-analysis likening the effect of all interventions in one single model ranks interventions containing an economic component followed by technical component with the best results. Educational or attitudinal components were ranked next, with standard care always considered the worst option. ${ }^{46}$ The GuildLink adherence intervention relies on educational components to enhance medication adherence. Moving forward incorporating attitudinal and technical components into this intervention would improve results. Evidence suggests that only $20 \%$ of the national health interventions produce sufficient savings to be at or near budget neutrality. ${ }^{47} \mathrm{~A}$ balance between intervention costs and achievable savings attributable to the intervention needs to be obtained. Financial incentives or rewards are not necessarily a viable long-term solution for 
a national health strategy. While exhibiting some success in improving processes in primary care, limited evidence of improved health outcomes or cost savings exists with interventions containing economic motivation, often eroding the potential economic gain. ${ }^{48-50}$ Medication adherence intervention programs represent an opportunistic national policy initiative to increase utilization of prescription medication to reduce the preventable burden currently placed on the Australian health care system. Pharmacists are ideally placed to implement, modify and sustain clearly defined adherence-enhancing interventions across a dynamic population. This way they can minimize negative therapeutic outcomes due to nonadherence, while increasing prescription volumes. ${ }^{51}$

The strategy developed to quantify the economic impact of medication non-adherence in Australia was conceptual. To date, there has been no specific estimate of the cost medication non-adherence poses to the Australian population. This study improves upon previous international estimates, despite its limitations. Actual medical condition prevalence estimates were applied from latest available population statistics. ${ }^{33,34}$ A national representative sample of non-adherent Australian adults were utilized to determine average medication adherence rates from prescription dispensing histories. Disease-specific per person cost of non-adherence was derived from the literature employing a "conservative" and "radical" approach. This analysis attempted to make estimates within a conceptual model that can be further tested and refined. Nonetheless, estimates from this study relied on data in the literature and on tertiary health statistics summaries. Moving forward the strategy should be tested in a real-life setting incorporating the determination of actual costs associated with this health problem. Prospective measurement of resource use in patients who receive the MedScreen Compliance intervention versus those who do not would address this. Furthermore, the current cost estimates only take into consideration three chronic conditions: hypertension, dyslipidemia and depression. For a more holistic outlook on the burden medication nonadherence places on the Australian health care system, additional conditions should be examined. Additionally, it would be beneficial to examine the offset of costs associated with delivering the service compared to costsaving potential. Currently, costs associated with delivering the service have not been considered. Despite these limitations, analysis of the economic impact of medication non-adherence across three highly prevalent conditions, utilizing real-life data demonstrated the positive impact pharmacist-led intervention can provide. Even when a conservative approach is employed.

\section{Conclusion}

Medication non-adherence across hypertension, dyslipidemia and depression costs $\$ 10.4$ billion annually, significantly contributing to avoidable health care costs in Australia. Community pharmacist-led medication adherence interventions have been demonstrated to improve adherence rates by $9.3 \%$ over a 12 -month period, reducing the burden incurred by non-adherence by $\$ 1.9$ billion. Given these findings, policy and decision makers should consider funding medication adherence programs to improve patient health outcomes and save money. Actively encouraging medication adherence through pharmacist-led intervention should be a top priority.

\section{Data sharing statement}

All data are available in paper.

\section{Acknowledgments}

This research is supported by an Australian Government Research Training Program Scholarship (RLC). This research received no specific grant from any funding agency in the public, commercial or not-for-profit sectors.

\section{Author contributions}

RLC drafted the initial form and all revisions of this manuscript. All other authors (RLC, ATB, EW, BD, NVDL, SIB, and VGC) made significant contributions to the manuscript and read and modified the drafts. All authors contributed to data analysis, drafting and revising the article, gave final approval of the version to be published, and agree to be accountable for all aspects of the work.

\section{Disclosure}

Naomi Van der Linden currently works at AstraZeneca Netherlands. The authors report no other conflicts of interest in this work.

\section{References}

1. Sabaté E. Adherence to Long-Term Therapies: Evidence for Action. World Health Organization. Geneva, Switzerland; 2003.

2. Sokol MC, McGuigan KA, Verbrugge RR, Epstein RS. Impact of medication adherence on hospitalization risk and healthcare cost. Med Care. 2005;43:521-530. 
3. DiMatteo MR, Giordani PJ, Lepper HS, Croghan TW. Patient adherence and medical treatment outcomes: a meta-analysis. Med Care. 2002;40(9):794-811. doi:10.1097/01.MLR.0000024612.61915.2D

4. Aitken M, Valkova S. Avoidable Costs in US Healthcare: The $\$ 200$ Billion Opportunity from Using Medicines More Responsibly. IMS Institute for Healthcare Informatics. United States; 2013:20-21.

5. Aitken M, Gorokhovich L. Advancing the Responsible Use of Medicines: Applying Levers for Change. IMS Institute for Healthcare Informatics. United States; 2012.

6. Pittman DG, Chen W, Bowlin SJ, Foody JM. Adherence to statins, subsequent healthcare costs, and cardiovascular hospitalizations. Am J Cardiol. 2011;107(11):1662-1666. doi:10.1016/j.amjcard.2011.0 1.052

7. Iuga AO, McGuire MJ. Adherence and health care costs. Risk Manag Healthc Policy. 2014;7:35-44. doi:10.2147/RMHP.S19801

8. Egede LE, Gebregziabher M, Dismuke CE, et al. Medication nonadherence in diabetes: longitudinal effects on costs and potential cost savings from improvement. Diabetes Care. 2012;35(12):2533-2539. doi: $10.2337 / \mathrm{dc} 12-0572$

9. Cantrell CR, Eaddy MT, Shah MB, Regan TS, Sokol MC. Methods for evaluating patient adherence to antidepressant therapy: a real-world comparison of adherence and economic outcomes. Med Care. 2006;44 (4):300-303. doi:10.1097/01.mlr.0000204287.82701.9b

10. NEHI. Thinking outside the Pillbox: A System-Wide Approach to Improving Patient Medication Adherence for Chronic Disease. New England Health Care Institute. United States; 2009.

11. Cutler RL, Fernandez-Llimos F, Frommer M, Benrimoj C, GarciaCardenas V. Economic impact of medication non-adherence by disease groups: a systematic review. BMJ Open. 2018;8(1):e016982.

12. Roughead L, Semple S, Rosenfeld E. Literature review: medication safety in Australia. Australian Commission on Safety and Quality in Health Care. Sydney, Australia; 2013.

13. Australian Institute of Health and Welfare. Health expenditure Australia 2015-16. Health and welfare expenditure series. Cat. no. HWE 68. Vol. 58. Canberra: AIHW; 2017.

14. Ye S, Krupka D, Davidson K. Diagnosing medication non-adherence in a patient with myocardial infarction. Front Psychol. 2012;3:267. doi:10.3389/fpsyg.2012.00267

15. Laba T, Lung T, Jan S, et al. Medication non adherence in a cohort of chronically ill Australians. Aust J Gen Pract. 2018;47:556-562.

16. Australian Government Department of Health PBS. Compliance to Medicines Working Group (CMWG) Report to Pharmaceutical Benefits Advisory Committee (PBAC). 2016. Available from: http:// www.pbs.gov.au/info/publication/factsheets/shared/cmwg-report-topbac. Accessed January 27, 2019.

17. Zweifel P, Felder S, Meiers M. Ageing of population and health care expenditure: a red herring? Health Econ. 1999;8(6):485-496.

18. Australian Institute of Health and Welfare. Australia's health 2018. Australia's health series no. 16. AUS 221. Canberra: AIHW.

19. Krivoy A, Balicer RD, Feldman B, et al. Adherence to antidepressant therapy and mortality rates in ischaemic heart disease: cohort study. $\mathrm{Br}$ j psychiatry. 2015;206(4):297-301. doi:10.1192/bjp.bp.114.155820

20. Shoval G, Stubbs B, Balicer RD, et al. Low adherence to antidepressants is associated with increased mortality in Parkinson disease patients. Parkinsonism Relat Disord. 2017;43:92-96. doi:10.1016/j. parkreldis.2017.07.032

21. Chowdhury R, Khan H, Heydon E, et al. Adherence to cardiovascular therapy: a meta-analysis of prevalence and clinical consequences. Eur Heart J. 2013;34(38):2940-2948. doi:10.1093/eurheartj/eht295

22. Ford ES, Ajani UA, Croft JB, et al. Explaining the decrease in U.S. deaths from coronary disease, 1980-2000. $N$ Engl J Med. 2007;356(23):2388-2398. doi:10.1056/NEJMsa053935

23. Vik SA, Maxwell CJ, Hogan DB. Measurement, correlates, and health outcomes of medication adherence among seniors. Ann Pharmacother. 2004;38(2):303-312. doi:10.1345/aph.1D252
24. Raebel MA, Schmittdiel J, Karter AJ, Konieczny JL, Steiner JF. Standardizing terminology and definitions of medication adherence and persistence in research employing electronic databases. Med Care. 2013;51(8 Suppl 3):S11-S21. doi:10.1097/ MLR.0b013e31829b1d2a

25. Ho PM, Bryson CL, Rumsfeld JS. Medication adherence: its importance in cardiovascular outcomes. Circulation. 2009;119 (23):3028-3035. doi:10.1161/CIRCULATIONAHA.108.768986

26. Pringle J, Coley KC. Improving medication adherence: a framework for community pharmacy-based interventions. Integr Pharm Res Pract. 2015;4:175-183. doi:10.2147/IPRP.S93036

27. Nasseh K, Frazee SG, Visaria J, Vlahiotis A, Tian Y. Cost of medication nonadherence associated with diabetes, hypertension, and dyslipidemia. Am J Pharm Benefits. 2012;4(2):e41-e47.

28. GuildLink Pty. Ltd. GuildCare MedScreen Compliance Protocol. The Pharmacy Guild of Australia. Australia; 2015.

29. Torres-Robles A, Wiecek E, Cutler R, et al. Using dispensing data to evaluate adherence implementation rates in community pharmacy. Front Pharmacol. 2019;10:130. doi:10.3389/fphar.2019.00130

30. Sattler EL, Lee JS, Perri M 3rd. Medication (re)fill adherence measures derived from pharmacy claims data in older Americans: a review of the literature. Drugs Aging. 2013;30(6):383-399. doi:10.1007/s40266-013-0074-z

31. Pillittere-Dugan D, Nau DP, McDonough K, Pierre Z. Development and testing of performance measures for pharmacy services. $J \mathrm{Am}$ Pharm Assoc. 2009;49(2):212-219. doi:10.1331/JAPhA.2009.09012

32. Australian Bureau of Statistics. Australian Demographic Statistics. ABS. Canberra; 2018.

33. Australian Bureau of Statistics. National Health Survey 2014-15 Australia. ABS. Canberra; 2015.

34. Australian Bureau of Statistics. Australian Health Survey 2011/12. ABS. Canberra; 2012.

35. Australian Bureau of Statistics. Australian Social Trends: Population Growth: Australia's Child Population. ABS. Canberra; 1997. Available from: http://www.abs.gov.au/ AUSSTATS/abs@.nsf/2f762f95845417aeca25706c00834efa/ f757d567b9b9b9eeca2570ec001b074e!OpenDocument. Accessed September 11, 2018.

36. Shemilt I, Thomas J, Morciano M. A web-based tool for adjusting costs to a specific target currency and price year. Evidence Policy. 2010;6(1):51-59. doi:10.1332/174426410X482999

37. Cutler RL, van der Linden N, Benrimoj SI, Fernandez-Llimos F, Garcia-Cardenas V. An evidence based model to consolidate medication adherence cost estimation: the MACE framework in. J Comp Eff Res. In press 2019.

38. Independent Hospital Pricing Authority. National Hospital Cost Data Collection Cost Report: Round 20 Financial Year 2015-16. 2018.

39. PBS Information Management Section; Pricing and Policy Branch; Technology Assessment and Access Division. Expenditure and Prescriptions Twelve Months to 30 June 2017. Australian Government Department of Health. Australia; 2017.

40. Meichenbaum D, Turk DC. Facilitating Treatment Adherence: A Practitioner's Guidebook. Plenum Press. New York, United States; 1987.

41. Committee for Economic Development of Australia. Healthcare: Reform or ration. CEDA. Melbourne, Australia; 2013.

42. Australian Treasury SW. Australia to 2050: Future Challenges. Canberra: Commonwealth of Australia; 2010.

43. Australian Government Department of Health. Department of Health Annual Report 2016-17. Canberra, Australia: Commonwealth of Australia; 2017.

44. Roebuck MC, Liberman JN, Gemmill-Toyama M, Brennan TA. Medication adherence leads to lower health care use and costs despite increased drug spending. Health Aff. 2011;30(1):91-99. doi:10.1377/ hlthaff.2009.1087 
45. Milosavljevic A, Aspden T, Harrison J. Community pharmacist-led interventions and their impact on patients ' medication adherence and other health outcomes: a systematic review. Int J Pharm Pract. 2018;26(5):387-397. doi:10.1111/ijpp.12462

46. Tonin FS, Wiecek E, Torres-Robles A, et al. An innovative and comprehensive technique to evaluate different measures of medication adherence: the network meta-analysis. Res Soc Administrative Pharm. 2019;15(4):358-365. doi: 10.1016/j. sapharm.2018.05.010 .

47. Bott DM, Kapp MC, Johnson LB, Magno LM. Disease management for chronically ill beneficiaries in traditional medicare. Health Aff. 2009;28(1):86-98. doi:10.1377/hlthaff.28.1.86
48. Scott A, Connelly LB. Financial incentives and the health workforce. Aust health rev. 2011;35(3):273-277. doi:10.1071/AH10904

49. Flodgren G, Eccles MP, Shepperd S, Scott A, Parmelli E, Beyer FR. An overview of reviews evaluating the effectiveness of financial incentives in changing healthcare professional behaviours and patient outcomes. Cochrane Database Syst Rev. 2011;6 (7):CD009255. doi: 10.1002/14651858.CD009255

50. de Bruin SR, Baan CA, Struijs JN. Pay-for-performance in disease management: a systematic review of the literature. BMC Health Serv Res. 2011;11:272. doi:10.1186/1472-6963-11-272

51. Johnson JA, Bootman JL. Drug-related morbidity and mortality. A cost-of-illness model. Arch Intern Med. 1995;155(18):1949-1956.

\section{Publish your work in this journal}

Patient Preference and Adherence is an international, peer-reviewed, open access journal that focusing on the growing importance of patient preference and adherence throughout the therapeutic continuum. Patient satisfaction, acceptability, quality of life, compliance, persistence and their role in developing new therapeutic modalities and compounds to optimize clinical outcomes for existing disease states are major areas of interest for the journal. This journal has been accepted for indexing on PubMed Central. The manuscript management system is completely online and includes a very quick and fair peer-review system, which is all easy to use. Visit http:// www.dovepress.com/testimonials.php to read real quotes from published authors. 